\section{A black mildew fungus, Schiffnerula azadirachtae sp. nov. (Ascomycota: Englerulaceae) from Kerala, India}

\author{
V.B. Hosagoudar ${ }^{1} \&$ A. Sabeena ${ }^{2}$ \\ 1,2 Tropical Botanic Garden and Research Institute, Palode, \\ Thiruvananthapuram, Kerala 695562, India \\ Email: ${ }^{1}$ vbhosagoudar@rediffmail.com (corresponding author)
}

The backyard medicinal plant of Deccan region, Azadirachta indica A. Juss., extensively planted in Kerala State harbours several fungal parasites. During our study on foliicolous fungi of the region, we collected black mildew fungus on this plant. Critical microscopic examination of the fungus revealed that it is hitherto unrecorded species of the genus Schiffnerula.

\section{Schiffnerula azadirachtae sp. nov.} (Fig. 1)

Material examined: 10.viii.2010, on leaves of Azadiracta indica A. Juss. (Meliaceae), Kallara, Thiruvananthapuram, Kerala, India, coll. A. Sabeena, TBGT 4323 (holotype), part of the collection has been deposited in HCIO (MycoBank \# 561024).

Anamorph: Sarcinella azadirachtae Meenu, Sanjay K. Singh \& R.K. Chaudhary, J. Living World 1:107,

Date of publication (online): 26 March 2011

Date of publication (print): 26 March 2011

ISSN 0974-7907 (online) | 0974-7893 (print)

Editor: R.K. Verma

\section{Manuscript details:}

Ms \# 02584

Received 23 September 2010

Final received 06 March 2011

Finally accepted 09 March 2011

Citation: Hosagoudar, V.B. \& A. Sabeena (2011). A black mildew fungus Schiffnerula azadirachtae sp. nov. (Ascomycota: Englerulaceae) from Kerala, India. Journal of Threatened Taxa 3(3): 1620-1621.

Copyright: (C) V.B. Hosagoudar \& A. Sabeena 2011. Creative Commons Attribution 3.0 Unported License. JoTT allows unrestricted use of this article in any medium for non-profit purposes, reproduction and distribution by providing adequate credit to the authors and the source of publication.

Acknowledgements: We thank Dr. A. Subramoniam, Director, Tropical Botanic Garden and Research Institute, Palode, Thiruvananthapuram Kerala for the facilities.

OPEN ACCESS | FREE DOWNLOAD
1994.

Coloniae amphigenae, tenues, ad $2 \mathrm{~mm}$ diam., confluentes. Hyphae brunneae, flexuosae, opposite, alternate vel unilateralis acuteque vel laxe ramosae, laxe reticulatae, cellulae 12-35 x 4-6 $\mu \mathrm{m}$. Appressoria alternata vel unilateralis, globosa, integra, $7-10 \times 7-10 \mu \mathrm{m}$. Conidia Questieriella dispersa in coloniis, curvula, 3-septata, leniter constrictus ad septata, attenuatus ad ambi apicem, 27-40 x 7-10 $\mu \mathrm{m}$. Sarcinella conidiophora producentes hyphis lateralis, solitaris, recta, flexuosa, mononemata; cellulae conidiogenae terminalis, monoblasticae, integratae, cylindraceae. Conidia Sarcinella blastica, terminalis, plerumque sessilis, solitaria, ovata vel globosa, sarciniformes, 2-7 cellula, constrictus ad septatus, $27-42 \mu \mathrm{m}$ diam., parietus glabrus. Thyriothecia dispersa, ovata, orbicularis, cellulae peridiales radiatus ad initio, dissolutus ad centre ad maturitatatus et asci visa, ad $70 \mu \mathrm{m}$ diam., cellulae marginales radiatae; asci 2-4 per thyriotheciis, globosi, octospori, 12-20 $\mu \mathrm{m}$ diam.; ascosporae oblongae, conglobatae, uniseptatae, constrictus ad septatae, $15-20 \times 7-10 \mu \mathrm{m}$, parietus glabrus.

Colonies amphigenous, thin, up to $2 \mathrm{~mm}$ in diameter, confluent. Hyphae brown, flexuous, branching opposite, alternate to unilateral at acute to wide angles, loosely reticulate, cells $12-35$ x 4-6 $\mu \mathrm{m}$. Appressoria alternate to unilateral, globose, entire, $7-10 \times 7-10 \mu \mathrm{m}$. Conidia of Questieriella scattered in the colonies, curved, 3 -septate, slightly constricted at the septa, taper towards both ends, $27-40 \times 7-10$ $\mu \mathrm{m}$. Sarcinella conidiophores produced lateral to the hyphae, single, straight, flexuous, mononematous; conidiogenous cells terminal, monoblastic, integrated, cylindrical. Sarcinella conidia blastic, terminal, mostly sessile, solitary, ovate to globose, sarciniform, 2-7 celled, constricted at the septa, $27-42 \mu \mathrm{m}$ in diameter, wall smooth. Thyriothecia scattered, globose, ovate, orbicular, peridial cells initially radiating, later the central portion dissolved by exposing asci, up to $70 \mu \mathrm{m}$ in diameter, marginal cells radiating; asci $2-4$ per thyriothecia, octosporous, $12-20 \mu \mathrm{m}$ in diameter; ascospores oblong, conglobate, uniseptate, constricted at the septum, 15-20 x 7-10 $\mu \mathrm{m}$, wall smooth.

Meenu et al. (1994) have described Sarcinella azadirachtae on Azadiracta indica from Nepal. 


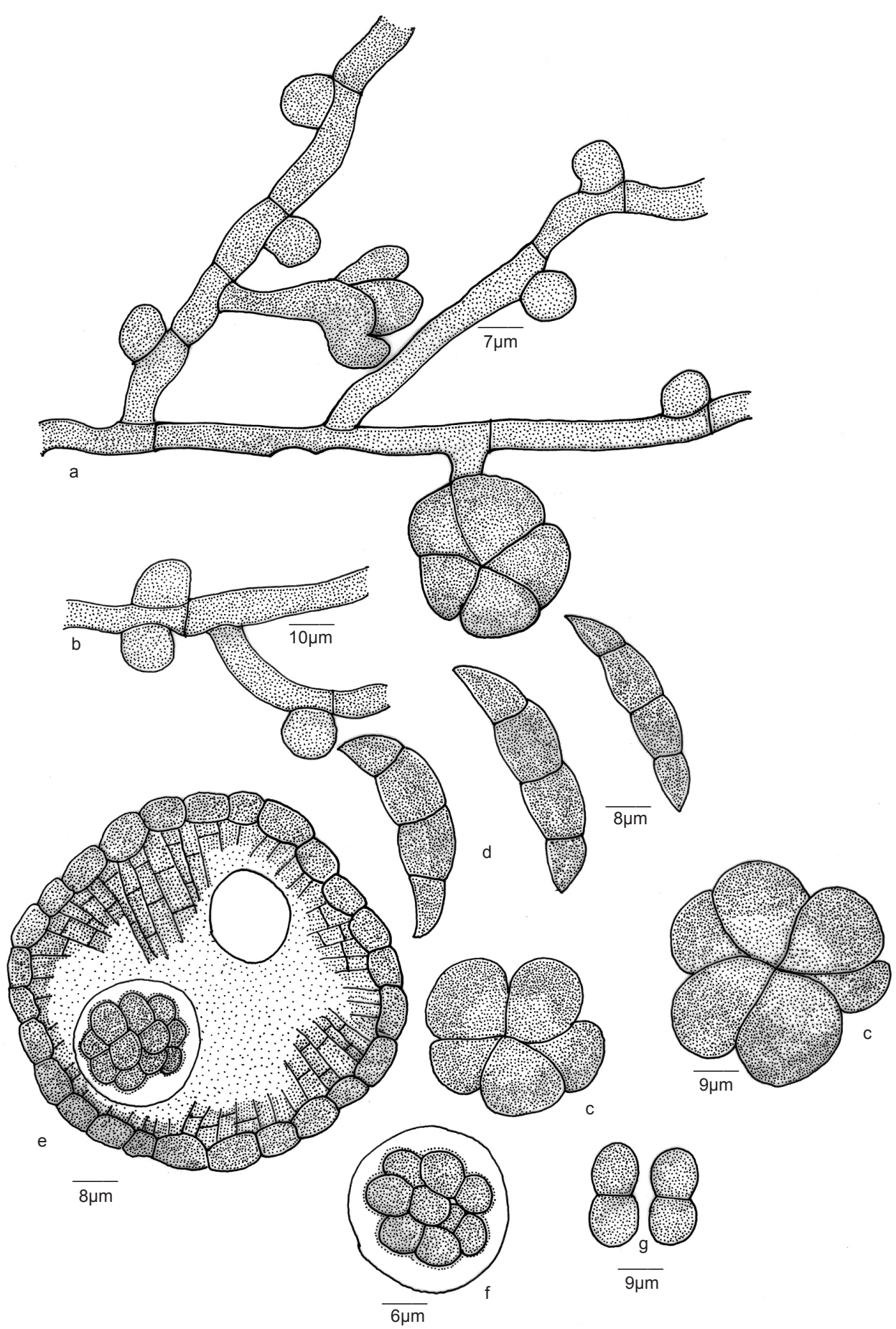

Figure 1. Schiffnerula azadirachtae sp. nov.

a - Appressoriate mycelium with developing and developed Sarcinella conidia; b - Opposite appressoria; c - Conidia of the form genus Sarcinella; $d$ - Conidia of the form genus Questieriella; e - Thyriothecium with exposed asci; $f$ - Ascus; $g$ - Ascospores

However, the teleomorph supersedes anamorph. Hence, it has been accommodated in a new species.

\section{REFERENCE}

Meenu, S.K. Singh \& R.K. Chaudhary (1994). New species and new records of Sarcinella from Nepal Himalayas. Journal of Living World 1(2): 106-111. 